\title{
Correction for Restriction of Range when Both $X$ and $Y$ are Truncated
}

\author{
Ralph A. Alexander, Kenneth P. Carson, George M. Alliger, \\ and Gerald V. Barrett \\ The University of Akron
}

The effect of range restriction on one variable in a bivariate normal distribution on the $X-Y$ correlation and the problem of estimating unrestricted from restricted correlations has been widely studied for more than half a century. The behavior of correction formulas under truncation of both $X$ and $Y$, however, remains largely unresearched. The performance of the correction formula for unidimensional truncation (Thorndike, 1947, Case 2) and an approximation procedure for correcting for bidimensional truncation proposed by Wells and Fruchter (1970) were investigated. The Thorndike correction formula undercorrects in most circumstances. The Wells and Fruchter procedure performs quite well under most conditions but often results in a slight overcorrection. The performance of the Wells and Fruchter and Thorndike formulas are also compared under truncation on $X$ or $Y$ alone. In these circumstances the Wells and Fruchter correction is either equal or markedly superior to the traditional correction. Based on overall performance in recapturing the unbiased population values under both unidimensional and bidimensional truncation, the Wells and Fruchter correction is recommended as the preferred procedure in many practical settings.

Restriction of range has been widely studied since Karl Pearson's (1903) initial work on the subject. Of particular interest here is the literature that addresses the problem of identifying changes in the correlation, $\rho$, of a bivariate normal population when one of the two variables is directly truncated or when the two variables are indirectly restricted by truncation on some third variable. Correction formulas for estimating unbiased $\rho$ appeared in the psychological literature by the early 1920's (Kelley, 1923; Otis, 1922), although Thorndike (1947) is often credited with bringing them into general use in the field of psychological testing and measurement. His Cases 1 and 2 deal with direct truncation and his Case 3 with indirect truncation. Recent research interest in this area has focused on the performance of these correction formulas (particularly Case 2) under various violations of the distribution assumptions or on the effects of applying the direct truncation correction formula when indirect truncation has occurred (Greener \& Osburn, 1979; Gross, 1982; Gross \& Fleischman, 1983; Linn, 1968).

Although the problem of unidimensional truncation (restriction on either $X$ or $Y$ ) has a long history of extensive research, the problem of truncation in both $X$ and $Y$ (bidimensional truncation) has been much more resistant to analysis, primarily because of the intractability of the mathematics. While Pearson (1908) presented a partial solution, only relatively recently has it been more completely solved (Birnbaum \& Meyer, 1953; Finney, 1962; Tallis, 1961). Formulas now exist for computing population means, 
variances, and correlations under truncation of both $X$ and $Y$, but explicit formulas for calculating unbiased from biased values have not yet been found (Regier \& Hamdan, 1971). Wells and Fruchter (1970) developed a procedure for approximating such corrected values. To date, both the usual correction formula (Thorndike's Case 2) and the Wells and Fruchter procedure remain largely unresearched as to their performance under truncation on both $X$ and $Y$. This article reports the accuracy of both the Thorndike and Wells and Fruchter correction formulas for estimating unbiased $\rho$ under both unidimensional and bidimensional truncation.

\section{Method}

Beginning from an unrestricted bivariate normal $X-Y$ distribution with known means, standard deviations, and correlation $\left(\mu_{x}, \mu_{y}, \sigma_{x}, \sigma_{y}, \rho\right)$ and cutting points $a$ on $X$ and $b$ on $Y$, biased parameters in the restricted distribution $\left(\mu_{x}^{\prime}, \mu_{y}^{\prime}, \sigma_{x}^{\prime}, \sigma_{y}^{\prime}, \rho^{\prime}\right)$ were computed. These values formed the basis for applying correction formulas to obtain estimates of the unrestricted correlation.

\section{Computation of Restricted Population Parameters}

Regier and Hamdan (1971) and Weiler (1959) provide mathematical expressions for calculating the parameters of a bivariate normal distribution truncated such that values outside the range $(a \leqslant x \leqslant \infty$, $b \leqslant y \leqslant \infty$ ) are excluded. Two familiar functions of any standardized univariate normal distribution $v$ with cutting point $t$ are:

$Z(t)=\left[1 /(2 \pi)^{1 / 2}\right] \exp \left[(-1 / 2) t^{2}\right]$,

$Q(t)=\int_{t}^{\infty} Z(v) d v$

Similarly, for any standardized bivariate normal distribution of $u, v$ with cutting points $s$ and $t$ :

$Z(s, t)=\left\{1 /\left[2 \pi\left(1-\rho^{2}\right)^{1 / 2}\right]\right\} \exp \left\{\left[-1 /\left(2-2 \rho^{2}\right)\right]\left(s^{2}-2 \rho s t+t^{2}\right)\right\} \quad$,

$Q(s, t)=\int_{s}^{\infty} \int_{t}^{\infty} Z(u, v) d u d v$

Finally, define

$A=(b-\rho a) /\left(1-\rho^{2}\right)^{1 / 2}$,

$B=(a-\rho b) /\left(1-\rho^{2}\right)^{1 / 2}$.

In the truncated distribution, then,

$$
\begin{aligned}
& E(X)=[Z(a) Q(A)+\rho Z(b) Q(B)] / Q(a, b), \\
& E(Y)=[Z(b) Q(B)+\rho Z(a) Q(A)] / Q(a, b), \\
& E\left(X^{2}\right)=\left[a Z(a) Q(A)+\rho^{2} b Z(b) Q(B)+\rho\left(1-\rho^{2}\right) Z(a, b)+Q(a, b)\right] / Q(a, b), \\
& E\left(Y^{2}\right)=\left[b Z(b) Q(B)+\rho^{2} a Z(a) Q(A)+\rho\left(1-\rho^{2}\right) Z(a, b)+Q(a, b)\right] / Q(a, b), \\
& E(X Y)=\left\{\rho[a Z(a) Q(A)+b Z(b) Q(B)+Q(a, b)]+\left(1-\rho^{2}\right) Z(a, b)\right\} / Q(a, b), \\
& \mu_{x}^{\prime}=E(X) ; \mu_{y}^{\prime}=E(Y), \\
& \sigma_{x}^{\prime}=\left\{E\left(X^{2}\right)-[E(X)]^{2}\right\}^{1 / 2} ; \quad \sigma_{y}^{\prime}=\left\{E\left(Y^{2}\right)-[E(Y)]^{2}\right\}^{1 / 2}, \\
& \text { and } \\
& \rho^{\prime}=\left[E(X Y)-\mu_{x}^{\prime} \mu_{y}^{\prime}\right] / \sigma_{x}^{\prime} \sigma_{y}^{\prime} .
\end{aligned}
$$




\section{Restriction of Range Corrections}

In the present notation, the Thorndike (1947) Case 2 correction formula is given by $\rho_{T}=\rho^{\prime} /\left[\rho^{\prime 2}+\left(\sigma_{x}^{\prime} / \sigma_{x}\right)^{2}\left(1-\rho^{\prime 2}\right)\right]^{1 / 2}$.

Wells and Fruchter reasoned that under direct truncation on both $X$ and $Y, \sigma_{x}^{\prime}$ and $\sigma_{y}^{\prime}$ (restricted standard deviations) deviate from their respective unrestricted values $\left(\sigma_{x}, \sigma_{y}\right)$ as a result of both direct and indirect truncation. That is, $\sigma_{x}^{\prime}$ as a function of $\sigma_{x}$ is influenced directly by the truncation on $X$ and indirectly by the truncation on $Y$. Similarly for $\sigma_{y}^{\prime}$. Their procedure attempts to partition the changes from unrestricted to restricted standard deviations into components due to direct and indirect truncation. Putting their Equations 11 through 17 into the present notation, that procedure is as follows:

$$
\begin{aligned}
& \rho_{1}=\rho^{\prime} /\left[\rho^{\prime 2}+\left(\sigma_{x}^{\prime} / \sigma_{x}\right)^{2}\left(1-\rho^{\prime 2}\right)\right]^{1 / 2}, \\
& \sigma_{y 1}=\sigma_{y}\left[\left(1-\rho_{1}^{2}\right) /\left(1-\rho^{\prime 2}\right)\right]^{1 / 2}, \\
& \sigma_{y 2}=\sigma_{y}+\sigma_{y}^{\prime}-\sigma_{y 1}, \\
& \rho_{2}=\rho^{\prime} /\left[\rho^{\prime 2}+\left(\sigma_{y 2} / \sigma_{y}\right)^{2}\left(1-\rho^{\prime 2}\right)\right]^{1 / 2}, \\
& \sigma_{x 1}=\sigma_{x}\left[\left(1-\rho_{2}^{2}\right) /\left(1-\rho^{\prime 2}\right)\right]^{1 / 2}, \\
& \sigma_{x 2}=\sigma_{x}+\sigma_{x}^{\prime}-\sigma_{x 1}, \\
& \text { and } \\
& \rho_{1}=\rho^{\prime} /\left[\rho^{\prime 2}+\left(\sigma_{x 2} / \sigma_{x}\right)^{2}\left(1-\rho^{\prime 2}\right)\right]^{1 / 2} .
\end{aligned}
$$

After the first pass through Equations 16 through 22, additional iterations of Equations 17 through 22 are calculated until the results converge on stable values of $\sigma_{x 2}$ and $\sigma_{y 2}$. Once stability has been reached, compute

$\rho_{W F}=\rho_{1} /\left[\rho_{1}^{2}+\left(\sigma_{y 2} / \sigma_{y}\right)^{2}\left(1-\rho_{1}^{2}\right)\right]^{1 / 2}$.

\section{Data Analysis}

For the analysis under bidimensional truncation, unrestricted standardized bivariate normal populations were defined $\left(\mu_{x}=\mu_{y}=0 ; \sigma_{x}=\sigma_{y}=1\right)$ for values of $\rho$ from -.90 to +.90 in increments of .10. Cutting points $a$ on $X$ and $b$ on $Y$ were varied from $-2.0 \sigma$ to $+2.0 \sigma$ at $.2 \sigma$ intervals.

Since all computations were carried out by computer, the partial area under a unidimensional normal curve (Equation 2) was found using the IMSL subroutine MDNOR and the partial volume of a bivariate normal distribution (Equation 4) was found using IMSL subroutine MDBNOR (IMSL, 1979). The remaining Equations 1 through 14 were used to calculate $\mu_{x}^{\prime}, \mu_{y}^{\prime}, \sigma_{x}^{\prime}, \sigma_{y}^{\prime}$, and $\rho^{\prime}$ for each of the 8,379 combinations of $a, b$, and $\rho$ (21 values of $a$ by 21 values of $b$ by 19 values of $\rho$ ). In order to check the accuracy of the computing algorithms, computed values of $\rho^{\prime}$ were compared with tabled values in Regier and Hamdan (1971) and values of $\sigma^{\prime}$ and $\mu^{\prime}$ with values from Weiler (1959).

Range restriction corrected estimates of $\rho$ were computed as if unidimensional truncation had occurred on $X$ using the Thorndike correction ( $\rho_{T}$, Equation 15). Equations 16 through 23 were used to compute the Wells and Fruchter approximation $\left(\rho_{W F}\right)$ at one to nine iterations.

The percentage error that would result in using the corrected correlation to estimate the unrestricted correlation was calculated by (for example)

$$
P E_{T}=\left[\left(\rho_{T}-\rho\right) / \rho\right] 100 \text {. }
$$

The sign of this percent error value provides the direction of error with positive values indicating overcorrection and negative values indicating undercorrection. 
Finally, the investigation of unidimensional truncation involved repeating the analyses just described for $a=-12.0 \sigma$ (i.e., effectively setting the cutting score of $X$ at $-\infty$ ) and again for $b=-12.0 \sigma$ (Weiler, 1959).

\section{Results}

\section{Bidimensional Truncation}

In order to summarize the overall performance of the Thorndike (Case 2) and Wells and Fruchter procedures for range restriction correction under conditions of truncation on both $X$ and $Y$, the percentage of error (Equation 24) was grouped into 10 categories: $-25 \%$ or more, $-10 \%$ to $-25 \%,-5 \%$ to $-10 \%,-1 \%$ to $-5 \%, 0 \%$ to $-1 \%, 0 \%$ to $+1 \%,+1 \%$ to $+5 \%,+5 \%$ to $+10 \%,+10 \%$ to $+25 \%$, and $+25 \%$ or more. For each value of unbiased $\rho$, a particular correction was applied to the 441 values of biased $\rho$ ( 21 cuts on $X$ by 21 cuts on $Y$ ). These 441 values were grouped by percentage error category. The frequency of these error categories as a function of the unrestricted correlation ( $\rho$ ) are presented in Table 1 for the Thorndike Case 2 correction, and in Table 2 for the Wells and Fruchter correction without iteration.

Before discussing these results, two comments are in order. First, since truncation will leave an unrestricted zero correlation $(\rho=0)$ essentially unchanged, the tables do not include values for this case. Second, for increasingly large negative correlations, more severe restriction of range (large positive value of $a$ and $b$ ) will result in excessively small remaining partial volumes (Equation 4). In these cases, computations cannot be carried out with sufficient accuracy to provide meaningful results and the values were excluded from computation (cf. Regier \& Hamdan, 1971, Table 1). As a result, the frequencies of percentage error in Tables 1 and 2 for negative $\rho \geqslant .20$ will sum to less than 441 .

In their 1970 article, Wells and Fruchter reasoned (but presented no data) that the Thorndike formula would overcorrect. From Table 1 it can be seen that use of the Thorndike Case 2 correction formula under bidimensional truncation results in an undercorrection the majority of the time. Only in the case of $\rho \geqslant .70$ is overcorrection found, and then the overcorrection never exceeds $1 \%$. On the other hand, the extent of undercorrection can be substantial, often $-25 \%$ or more. Table 2 shows that the Wells and Fruchter correction performs reasonably well even without iteration. In fact, in the large majority of cases, the percentage of error is within $\pm 5 \%$.

In their own investigation of the correction procedure, Wells and Fruchter found that the estimations of $\sigma_{x}$ and $\sigma_{y}$ would tend to stabilize at four to five iterations and thus recommended four or five iterations in order to obtain the most accurate estimates of the unrestricted correlation. Comparison of the present results from up to nine iterations found that, for all negative correlations and for positive $\rho \leqslant .40$, application of their procedure without iteration and at five iterations produces virtually identical results. For positive correlations of .50 or greater, though the procedure at five iterations gives somewhat more accurate results, the procedure without iteration is somewhat more conservative-less likely to overcorrect.

Tables 3 and 4 give a more specific picture of the nature and extent of both the restricted and corrected values of correlations under bidimensional truncation. The restricted correlations, corrected values resulting from application of the Thorndike formula, and corrected correlations from the Wells and Fruchter procedure without iteration are presented for $\rho=.2$ in Table 3 and for $\rho=.6$ in Table 4 . In the interest of conserving space, Tables 3 and 4 show values for cuts on $X$ and $Y$ from $-2.0 \sigma$ to $+2.0 \sigma$ in increments of $.4 \sigma$.

Inspection of Tables 3 and 4 clearly shows the pattern of accuracy in using the Thorndike correction under bidimensional truncation. This correction formula most accurately recaptures the unrestricted correlation when restriction on $Y$ is negligible and restriction on $X$ is substantial ( $Y$ cut $=-2.0 \sigma, X$ cut 
Table 1

Frequency of Ranges of Percent Error $\left(P E_{T}\right)$ Using the

Thorndike Correction to Estimate $p$

\begin{tabular}{|c|c|c|c|c|c|c|c|c|c|c|}
\hline \multirow[b]{4}{*}{$\underset{\rho}{\text { Unrestricted }}$} & \multicolumn{9}{|c|}{ Range of PE } & \\
\hline & \multicolumn{5}{|c|}{ Undercorrection } & \multicolumn{5}{|c|}{ overcorrection } \\
\hline & $-25 \%$ & $-10 \%$ & $-5 \%$ & $-1 \%$ & 0 & 0 & $+1 \%$ & $+5 \%$ & $+10 \%$ & $+25 \%$ \\
\hline & $\begin{array}{l}\text { or } \\
\text { More }\end{array}$ & $\begin{array}{l}\text { to } \\
-25 \%\end{array}$ & $\begin{array}{l}\text { to } \\
-10 \%\end{array}$ & $\begin{array}{l}\text { to } \\
-5 \%\end{array}$ & $\begin{array}{l}\text { to } \\
-1 \%\end{array}$ & $\begin{array}{l}\text { to } \\
+1 \%\end{array}$ & $\begin{array}{l}\text { to } \\
+5 \%\end{array}$ & $\begin{array}{l}\text { to } \\
\text { +10\% }\end{array}$ & $\begin{array}{l}\text { to } \\
+25 \%\end{array}$ & $\begin{array}{l}\text { or } \\
\text { More }\end{array}$ \\
\hline+.90 & 0 & 30 & 75 & 98 & 87 & 151 & 0 & 0 & 0 & 0 \\
\hline+.80 & 36 & 109 & 51 & 71 & 82 & 92 & 0 & 0 & 0 & 0 \\
\hline+.70 & 101 & 99 & 45 & 72 & 97 & 27 & 0 & 0 & 0 & 0 \\
\hline+.60 & 150 & 93 & 46 & 72 & 80 & 0 & 0 & 0 & 0 & 0 \\
\hline+.50 & 185 & 93 & 45 & 72 & 46 & 0 & 0 & 0 & 0 & 0 \\
\hline+.40 & 215 & 93 & 45 & 66 & 22 & 0 & 0 & 0 & 0 & 0 \\
\hline+.30 & 242 & 92 & 47 & 56 & 4 & 0 & 0 & 0 & 0 & 0 \\
\hline+.20 & 266 & 91 & 46 & 38 & 0 & 0 & 0 & 0 & 0 & 0 \\
\hline+.10 & 285 & 90 & 50 & 16 & 0 & 0 & 0 & 0 & 0 & 0 \\
\hline-.10 & 310 & 93 & 36 & 2 & 0 & 0 & 0 & 0 & 0 & 0 \\
\hline$-.20 \star$ & 319 & 95 & 25 & 0 & 0 & 0 & 0 & 0 & 0 & 0 \\
\hline$-.30^{\star}$ & 327 & 89 & 20 & 0 & 0 & 0 & 0 & 0 & 0 & 0 \\
\hline$-.40 \star$ & 331 & 84 & 20 & 1 & 0 & 0 & 0 & 0 & 0 & 0 \\
\hline$-.50 \star$ & 329 & 82 & 21 & 4 & 0 & 0 & 0 & 0 & 0 & 0 \\
\hline$-.60 \star$ & 315 & 81 & 25 & 9 & 0 & 0 & 0 & 0 & 0 & 0 \\
\hline$-.70^{\star}$ & 298 & 88 & 29 & 20 & 0 & 0 & 0 & 0 & 0 & 0 \\
\hline$-.80^{\star}$ & 254 & 90 & 38 & 45 & 5 & 0 & 0 & 0 & 0 & 0 \\
\hline$-.90 \star$ & 133 & 98 & 50 & 74 & 70 & 0 & 0 & 0 & 0 & 0 \\
\hline
\end{tabular}

Note. For each $\rho, 441$ values were calculated (21 values of a by 21 values of $b$ )

*These rows will not sum to 441 (see note above) since $\rho^{\prime}$ cannot be calculated with sufficient accuracy in cases of extreme restriction.

$=+2.0 \sigma)$. This correction becomes less and less accurate as restriction on $X$ becomes less severe and as truncation on $Y$ becomes more severe. This pattern is as would be expected since the Thorndike formula uses only the change in $\sigma_{x}$ to correct for restriction of range.

The pattern of results for the Wells and Fruchter procedure (without iteration) is quite different but similarly systematic. Referring to Table 2 (which provides the frequencies) and to Tables 3 and 4 (particularly Table 4), it is found that for relatively large correlations the corrected values are most often moderate overestimates, with the exception of moderate truncation on $X$ coupled with more severe truncation on $Y$ in which case the correction results in an underestimate. In the case of small correlations, the same pattern holds, except that the frequency of undercorrection becomes quite small and the degree of overcorrection increases.

\section{Unidimensional Truncation}

While the results just described provide support for the use of the Wells and Fruchter correction procedure when restriction of range occurs on both variables, the question remains as to the severity of error in using that procedure when only one of the two variables is truncated. 
Table 2

Frequency of Ranges of Percent Error ( $P E_{W F}$ ) Using the Wells and

Fruchter Correction Without Iteration to Estimate $\mathrm{P}$

\begin{tabular}{|c|c|c|c|c|c|c|c|c|c|c|}
\hline \multirow[b]{4}{*}{$\underset{\rho}{\text { Unrestricted }}$} & \multicolumn{10}{|c|}{ Range of $P E$} \\
\hline & \multicolumn{5}{|c|}{ Undercorrection } & \multicolumn{5}{|c|}{ Overcorrection } \\
\hline & $-25 \%$ & $-10 \%$ & $-5 \%$ & $-1 \%$ & 0 & 0 & $+1 \%$ & $+5 \%$ & $+10 \%$ & $+25 \%$ \\
\hline & $\begin{array}{l}\text { or } \\
\text { More }\end{array}$ & $\begin{array}{l}\text { to } \\
-25 \%\end{array}$ & $\begin{array}{l}\text { to } \\
-10 \%\end{array}$ & $\begin{array}{l}\text { to } \\
-5 \%\end{array}$ & $\begin{array}{l}\text { to } \\
-1 \%\end{array}$ & $\begin{array}{l}\text { to } \\
+1 \%\end{array}$ & $\begin{array}{l}\text { to } \\
+5\end{array}$ & $\begin{array}{l}\text { to } \\
+10 \%\end{array}$ & $\begin{array}{l}\text { to } \\
+25 \%\end{array}$ & $\begin{array}{l}\text { or } \\
\text { More }\end{array}$ \\
\hline+.90 & 0 & 0 & 57 & 135 & 91 & 158 & 0 & 0 & 0 & 0 \\
\hline 80 & 0 & 0 & 40 & 161 & 61 & 179 & 0 & 0 & 0 & 0 \\
\hline+.70 & 0 & 0 & 0 & 134 & 54 & 194 & 59 & 0 & 0 & 0 \\
\hline+.60 & 0 & 0 & 0 & 65 & 38 & 142 & 196 & 0 & 0 & 0 \\
\hline+.50 & 0 & 0 & 0 & 0 & 46 & 109 & 237 & 49 & 0 & 0 \\
\hline+.40 & 0 & 0 & 0 & 0 & 9 & 88 & 262 & 82 & 0 & 0 \\
\hline+.30 & 0 & 0 & 0 & 0 & 1 & 70 & 312 & 58 & 0 & 0 \\
\hline+.20 & 0 & 3 & 2 & 3 & 0 & 69 & 353 & 11 & 0 & 0 \\
\hline+.10 & 5 & 3 & 3 & 1 & 0 & 158 & 259 & 10 & 2 & 0 \\
\hline-.10 & 1 & 8 & 8 & 266 & 135 & 4 & 4 & 2 & 3 & 10 \\
\hline$-.20 \star$ & 7 & 4 & 8 & 378 & 23 & 2 & 2 & 3 & 4 & 8 \\
\hline$-.30 \star$ & 5 & 6 & 6 & 387 & 12 & 0 & 8 & 4 & 3 & 5 \\
\hline$-.40 \star$ & 7 & 4 & 13 & 374 & 13 & 3 & 8 & 0 & 6 & 8 \\
\hline$-.50 \star$ & 14 & 0 & 85 & 299 & 10 & 0 & 7 & 8 & 2 & 11 \\
\hline$-.60 \star$ & 17 & 2 & 140 & 224 & 18 & 10 & 2 & 8 & 7 & 2 \\
\hline$-.70^{\star}$ & 31 & 7 & 172 & 184 & 14 & 6 & 7 & 6 & 8 & 0 \\
\hline$-.80 \star$ & 44 & 5 & 201 & 157 & 19 & 2 & 4 & 0 & 0 & 0 \\
\hline$-.90^{\star}$ & 31 & 10 & 188 & 119 & 69 & 7 & 1 & 0 & 0 & 0 \\
\hline
\end{tabular}

Note. For each $\rho, 441$ values were calculated ( 21 values of $\underline{a}$ by 21 values of $\underline{b}$ )

*These rows will not sum to 441 (see note abqve) since $\rho$ ' cannot be calculated with sufficient accuracy in cases of extreme restriction.

Two possible situations can be distinguished: (1) the restriction is on $X$ and correctly diagnosed as such, and (2) the restriction is on $Y$ and improperly assumed to have been on $X$. In the first case, applications of the two correction formulas to correlations arising from restriction on $X$ only, show that both result in identical values for the corrected $\rho$ s.

In the situation where restriction has occurred on $Y$ but the Thorndike and Wells and Fruchter corrections have both been incorrectly applied (as though restriction had occurred on $X$ ), the Wells and Fruchter procedure is clearly superior. These results are summarized in Table 5 for positive $\rho$ and for restrictions on $Y$ at $-2.0 \sigma,-1.0 \sigma, \mu$, $+1.0 \sigma$, and $+2.0 \sigma$.

As can be seen from Table 5, the Wells and Fruchter procedure results in slight undercorrections with the largest underestimate for $\rho=.90$ and severe restriction on $Y(b=+2.0 \sigma)$. Also, the Wells and Fruchter procedure always results in less error in estimating the unrestricted $\rho$ than does the Thorndike Case 2 formula, often substantially so.

These results hold if the role of $X$ and $Y$ in the above discussion are reversed. That is, if the restriction is on $Y$ and correctly diagnosed, then the proper application of the Thorndike and of the Wells and 
Table 3

Restricted Population Coefficients $\left(\rho^{\prime}\right)$ and Estimated $\rho$ Using the Thorndike Restriction of Range Correction and the Wells and Fruchter Restriction of Range Correction for $\rho=.20$

\begin{tabular}{|c|c|c|c|c|c|c|c|c|c|c|c|}
\hline \multirow{2}{*}{$\begin{array}{c}\text { Cuts on } x \\
(\sigma)\end{array}$} & \multicolumn{11}{|c|}{ Cuts on $Y(\sigma)$} \\
\hline & -2.0 & -1.6 & -1.2 & -0.8 & -0.4 & $\frac{0.0}{0.0}$ & 0.4 & 0.8 & 1.2 & 1.6 & 2.0 \\
\hline \multicolumn{12}{|c|}{ Restricted Population Coefficients } \\
\hline ? & 179 & 171 & 160 & 147 & 132 & 118 & 105 & 094 & 084 & 075 & 068 \\
\hline & 171 & 164 & 153 & 141 & & 114 & 102 & 091 & 082 & 073 & 066 \\
\hline-1.2 & 160 & 153 & 144 & 133 & 121 & 109 & 097 & 087 & 078 & 070 & 064 \\
\hline-0.8 & 147 & 141 & 133 & 123 & 112 & 101 & 091 & 081 & 073 & 066 & 060 \\
\hline & 132 & 128 & 121 & 112 & 102 & 092 & 083 & 075 & 067 & 061 & 055 \\
\hline 0.0 & 118 & 114 & 109 & 101 & 092 & 084 & 075 & 068 & 061 & 055 & 051 \\
\hline 0.4 & 105 & 102 & 097 & 091 & 083 & 075 & 068 & 061 & 055 & 050 & 046 \\
\hline 0.8 & 094 & 091 & 087 & 081 & 075 & 068 & 061 & 055 & 050 & 045 & 042 \\
\hline 1.2 & 084 & 082 & 078 & 073 & & 061 & 055 & 050 & 045 & 040 & 037 \\
\hline 1.6 & 075 & 073 & 070 & 066 & 061 & 055 & 050 & 045 & 040 & 034 & 034 \\
\hline 2.0 & 068 & 066 & 064 & 060 & 055 & 051 & 046 & 042 & 037 & 034 & 031 \\
\hline \multicolumn{12}{|c|}{ Thorndike Corrected Coefficients } \\
\hline-2.0 & 190 & 182 & 170 & 155 & 140 & 125 & 111 & 0.99 & 088 & 078 & \\
\hline-1.6 & 191 & 182 & 171 & 157 & 141 & 126 & 112 & 099 & 088 & 079 & 071 \\
\hline-1.2 & 192 & 184 & 172 & 158 & 143 & 128 & 113 & 100 & 089 & 080 & 072 \\
\hline-0.8 & 192 & 185 & 174 & 160 & & 129 & 115 & 102 & & & 072 \\
\hline-0.4 & 193 & 186 & 175 & 162 & 147 & 131 & 116 & 103 & 092 & 082 & 073 \\
\hline & 194 & 187 & 177 & 164 & & 13 & 118 & 10 & 093 & 083 & \\
\hline & 194 & 188 & 179 & 166 & & 13 & 12 & 10 & 095 & & 075 \\
\hline 0.8 & 195 & 189 & 180 & 168 & 153 & 137 & 122 & 108 & 096 & 085 & 078 \\
\hline & 196 & 190 & 182 & 170 & & 140 & 125 & & & & \\
\hline 1.6 & 196 & 191 & 183 & 172 & 158 & 142 & 127 & 112 & 099 & 083 & 080 \\
\hline 2.0 & 197 & 192 & 185 & 174 & 160 & 145 & 129 & 116 & 103 & 092 & 082 \\
\hline \multicolumn{12}{|c|}{ Wells and Fruchter Corrected Coefficients } \\
\hline-2.0 & 201 & 201 & & 202 & 202 & 202 & 202 & 201 & 201 & 201 & 201 \\
\hline $\begin{array}{l}-1.0 \\
-1.12\end{array}$ & 201 & 202 & 202 & 203 & 2004 & 204 & 203 & 202 & 202 & $\begin{array}{l}20 L \\
203\end{array}$ & $\begin{array}{l}202 \\
203\end{array}$ \\
\hline-0.8 & 202 & 203 & 204 & 205 & 205 & 205 & 205 & 205 & 205 & 204 & 204 \\
\hline-0.4 & 202 & 203 & 204 & 205 & 20 & 206 & 20 & & & & 205 \\
\hline & 202 & 20 & 204 & 205 & & 207 & 20 & & & & 207 \\
\hline 0.4 & 202 & 203 & 204 & 205 & 20 & 207 & 207 & 207 & 208 & 207 & 207 \\
\hline 0.8 & 201 & 20 & 204 & 205 & 20 & 20 & 20 & & & & 210 \\
\hline 1.2 & 201 & 20 & 204 & 205 & 20 & 20 & 28 & & & & 210 \\
\hline 1.6 & 201 & 202 & 203 & 204 & 20 & 207 & 207 & 208 & 207 & 210 & 211 \\
\hline 2.0 & 201 & 202 & 203 & 204 & $20 !$ & 207 & 207 & 210 & 210 & 211 & 2. \\
\hline
\end{tabular}

Note. Decimal points omitted.

Fruchter procedures (by reversing the roles of $X$ and $Y$ in Equations 16 through 23) will result in identical corrected values. In summary, the proper diagnosis of unidimensional truncation and corrections applied correctly by the two methods will yield identical results. On the other hand, improper diagnosis (i.e., restriction is thought to have occurred on $X$ but actually occurred on $Y$ or vice versa) will result in the Wells and Fruchter correction being superior to the Thorndike correction. 
Table 4

Restricted Population Coefficients $\left(\rho^{\prime}\right)$ and Estimated $\rho$ Using the Thorndike Restriction of Range Correction and the Wells and Fruchter Restriction of Range Correction for $p=.60$

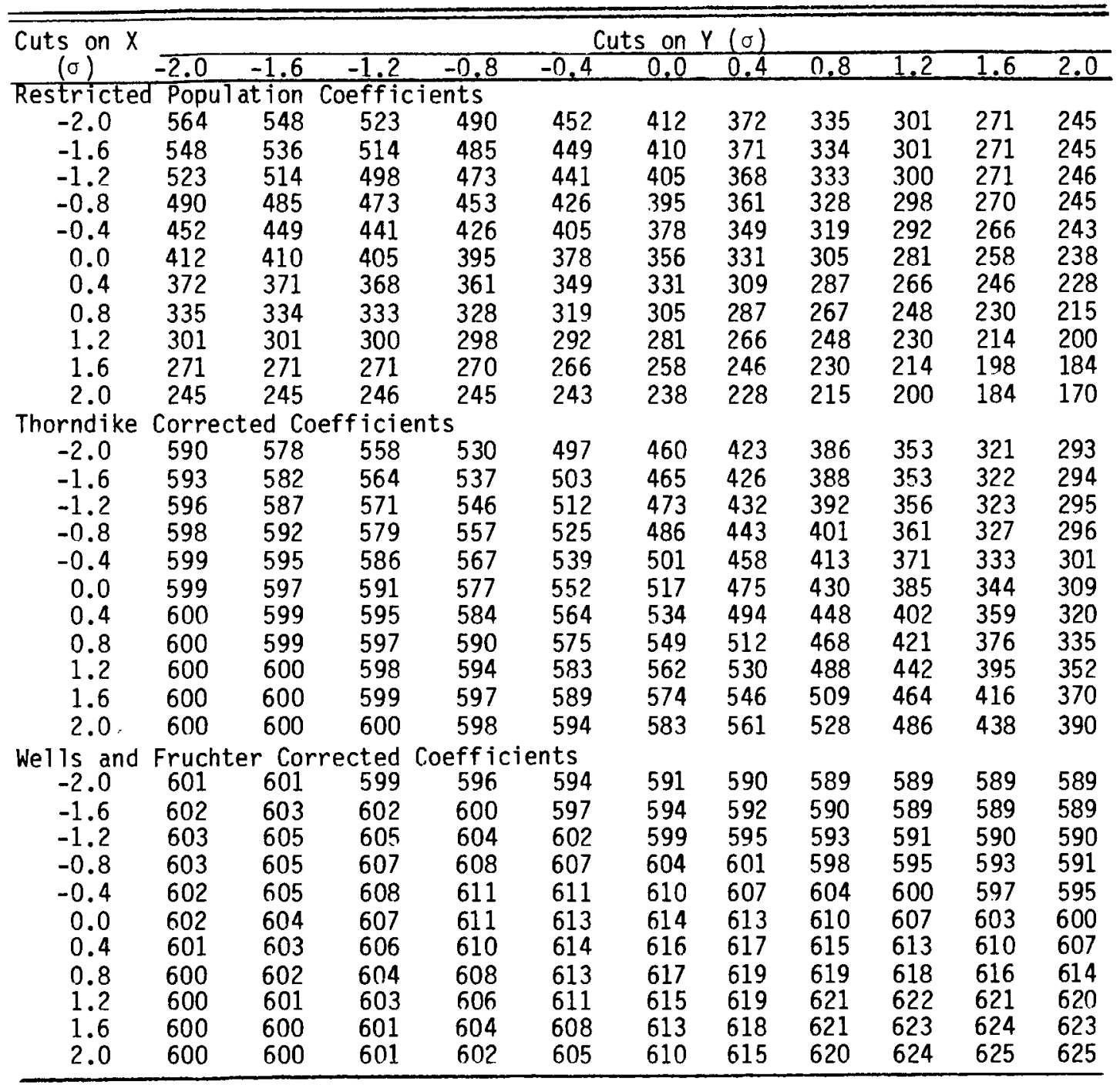

Note. Decimal points omitted.

\section{Discussion}

Before proceeding, a point of clarification may be needed. Throughout this article, the Thorndike Case 2 correction is referred to primarily. It should be kept in mind that the results described are identical for his Case 1 correction since the only difference between them has to do with whether knowledge of the unrestricted variance is available on the directly (Case 2) or indirectly (Case 1) truncated variable. 
Table 5

Estimated $\rho$ Using the Thorndike Correction $\left(\rho_{T}\right)$ and Wells and Fruchter Correction Without Iteration $\left(\rho_{W F}\right)$ for Range Restriction on $Y$ Only but Corrected Assuming Restriction on $X$ Only, or Restricted on $X$ only but Corrected Assuming Restriction on $Y$ Only.

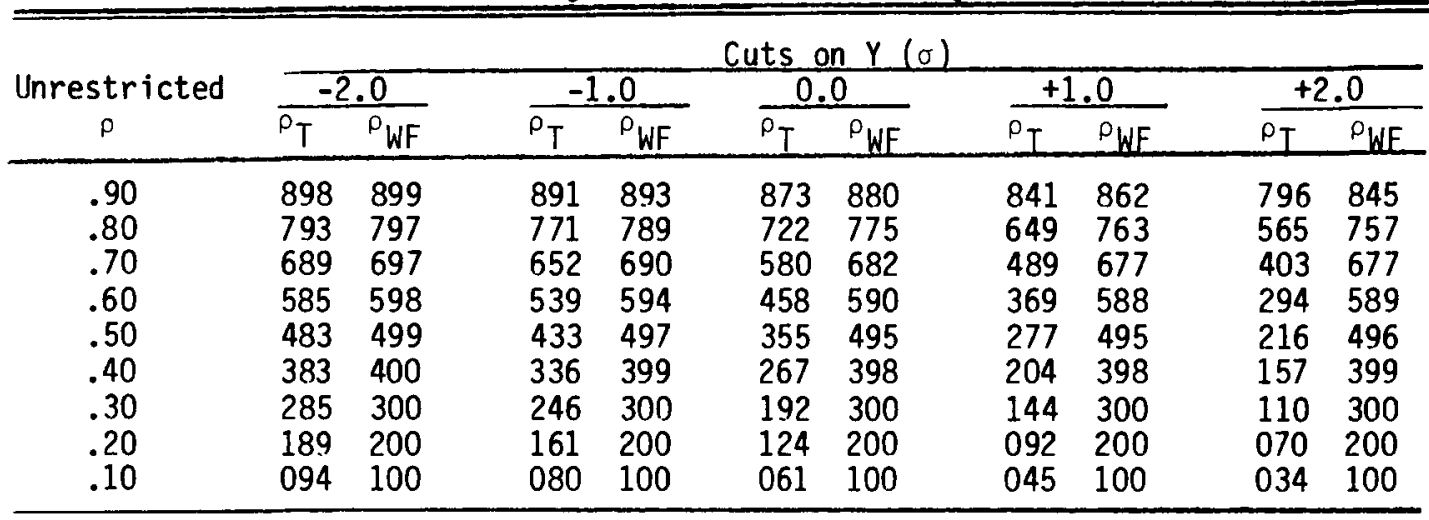

Note. $\rho_{T}$ and $\rho_{W F}$ are given to three decimal places, with the decimal point omitted in the table.

Research on restriction of range, the change from unrestricted to restricted correlations, and the performance of correction formulas has been confined, until recently, almost entirely to the case either of direct truncation on one (but not both) of the variables of interest or to restriction on some third (indirect) variable. Unidimensional truncation has been assumed in two practical measurement settings where bidimensional truncation is a more likely assumption.

The first measurement setting relates to reliability. Lord and Novick (1968) discussed the effects of relative homogeneity/heterogeneity of the sample on empirical estimates of reliability. Their discussion was based on the mathematics of direct unidimensional truncation. A moment's reflection, however, would suggest that in a test-retest or parallel forms reliability setting, restriction of range on " $X$ ", necessarily implies a similar restriction on " $Y$." Thus, reliability coefficients in such instances, if corrected using the Thorndike correction formula, may still be inappropriately low.

A second practical situation is that of predictive validation studies. In most such cases, it is assumed that $X$ (the predictor) is restricted due to explicit selection. In practice, not all of those admitted by the selection procedure remain available for measurement on the criterion. At the very least (since prediction is imperfect and is likely to result in some number of false positives), a subset of those will have left the organization due to failure to achieve some minimal level of success (Barrett, Phillips, \& Alexander, 1981).

These results suggest several areas for further research. The present study has dealt with the characteristics of correction formulas for population values. The relative sampling behavior of these corrections remains to be investigated. Since little research has been done on the distribution of sample $r$ s that have arisen from truncated populations (Alexander, Carson, Alliger, \& Barrett, 1983; Gajjar \& Subrahmaniam, 1978), and even less on the sampling distribution of corrected $r$ s (Gullikson \& Hopkins, 1976), generalization from the present results to sample values is necessarily tenuous. In addition, the methods of 
this study assume strict truncation on the population: the same assumption made by all restriction of range research. The behavior of biased correlations and correction formulas under violation of that assumption are still largely a matter of conjecture (Alexander, Barrett, Alliger, \& Carson, 1983).

Based on these results, it is concluded that in situations where the possibility of bidimensional truncation is suspected, the correction procedure of choice appears to be that of Wells and Fruchter. In addition, if the Wells and Fruchter procedure is applied when the situation is actually one of unidimensional truncation, then it will, at worst, equal the Thorndike result; and, if the wrong variable is assumed to be truncated, then it will perform better than the Thorndike correction.

\section{Refei"ences}

Alexander, R. A., Barrett, G. V., Alliger, G. M., \& Carson, K. P. (1983). Toward a general model of nonrandom sampling and the impact on population correlation: Generalization of Berkson's Fallacy and restriction of range. Manuscript submitted for publication.

Alexander, R. A., Carson, K. P., Alliger, G. M., \& Barrett, G. V. (1983). Further consideration of the power to detect non-zero validity coefficients under range restriction. Manuscript submitted for publication.

Barrett, G. V., Phillips, J. S., \& Alexander, R. A. (1981). Concurrent and predictive validity designs: A critical reanalysis. Journal of Applied Psychology, 66, 1-6.

Birnbaum, Z. W., \& Meyer, P. L. (1953). On the effect of truncation in some or all coordinates of a multinormal population. Journal of the Indian Society of Agricultural Statistics, 5, 17-28.

Finney, D. J. (1962). Cumulants of truncated multinormal distributions. Journal of the Royal Statistical Society, Series B, 24, 535-536.

Gajjar, A. V., \& Subrahmaniam, K. (1978). On the sample correlation coefficient in the truncated bivariate normal population. Communication in Statistics, Series $B, 7,455-477$.

Greener, J. M., \& Osburn, H. G. (1979). An empirical study of the accuracy of corrections for restriction of range due to explicit selection. Applied Psychological Measurement, 3, 31-41.

Gross, A. L. (1982). Relaxing assumptions underlying corrections for restriction of range. Educational and Psychological Measurement, 42, 795-801.

Gross, A. L., \& Fleischman, L. (1983). Restriction of range corrections when both distribution and selection assumptions are violated. Applied Psychological Measurement, 7, 227-237.

Gullikson, A., \& Hopkins, K. (1976). Interval estimation of correlation coefficients corrected for restriction of range. Educational and Psychological Measurement, 36, 9-25.
IMSL Library. (1979). Houston TX: International Mathematical and Statistical Libraries.

Kelley, T. L. (1923). Statistical method. New York: McMillan.

Linn, R. L. (1968). Range restriction problems in the use of self-selected groups for test validation. Psychological Bulletin, 69, 69-73.

Lord, F. M., \& Novick, M. R. (1968). Statistical theories of mental test scores. Reading MA: AddisonWesley.

Otis, A. A. (1922). A method of inferring the change in a coefficient of correlation resulting from a change in the heterogeneity of the group. Journal of Educational Psychology, 13, 293-294.

Pearson, K. (1903). Mathematical contributions to the theory of evolution-XI. On the influence of natural selection on the variability and correlation of organs. Philosophical Transactions of the Royal Society, London, Series A, 200, 1-66.

Pearson, K. (1908). On the influence of double selection on the variation and correlation of two characters. Biometrika, 6, 111-112.

Regier, M. H., \& Hamdan, M. A. (1971). Correlation in a bivariate normal distribution with truncation in both variables. Australian Journal of Statistics, 13, $77-82$.

Tallis, G. M. (1961). The moment generating function of the truncated multi-normal distribution. Journal of the Royal Statistical Society, Series B, 23, 223-229.

Thorndike, R. L. (1947). Research problems and techniques. (Report No. 3). Washington DC: U.S. Government Printing Office.

Weiler, H. (1959). Means and standard deviations of a truncated normal bivariate distribution. Australian Journal of Statistics, 1, 73-81.

Wells, D. G., \& Fruchter, B. (1970). Correcting the correlation coefficient for explicit restriction on both variables. Educational and Psychological Measurement, 30, 925-934. 


\section{Author's Address}

Send requests for reprints or further information to Ralph A. Alexander, Department of Psychology, The University of Akron, Akron OH 44325, U.S.A. 